\title{
Trigonometric Parallax of RCW 122
}

\author{
Y. W. Wu ${ }^{1,2}$, Y. Xu ${ }^{1}$, K. M. Menten ${ }^{2}$, X. W. Zheng ${ }^{3}$ and M.J. Reid ${ }^{4}$ \\ ${ }^{1}$ Purple Mountain Observatory, Chinese Academy of Sciences, Nanjing 210008, \\ email: ywwu@pmo.ac.cn \\ ${ }^{2}$ Max-Planck-Institut für Radioastronomie, Auf dem Hügel 69, 53121 Bonn, Germany \\ ${ }^{3}$ School of Astronomy and Space sience, Nanjing University, Nanjing 210093, China \\ ${ }^{4}$ Harvard-Smithsonian Center for Astrophysics, 60 Garden Street, Cambridge, MA 02138, USA
}

\begin{abstract}
As a part of the BeSSeL (Bar and Spiral Structure Legacy) survey, we report a trigonometric parallax for the massive star-forming region G348.70-1.04. Its distance is $3.38_{-0.27}^{+0.33}$ $\mathrm{kpc}$, indicating that it is in the Scutum-Centaurus arm. Its proper motion is $-0.73 \pm 0.04$ mas $\mathrm{yr}^{-1}$ toward the east and $-2.83 \pm 0.50$ mas $\mathrm{yr}^{-1}$ toward the north.
\end{abstract}

Keywords. astrometry, masers, stars: formation, techniques: high angular resolution

\section{Introduction}

The BeSSeL survey is a VLBA Key Science Project that will determine proper motions and parallaxes for methanol and water masers in several hundred massive star forming regions in the Galaxy. This project will allow us to locate spiral arms and determine the 3D motions of massive star-forming regions in our Galaxy. Currently, we are observing water and methanol masers towards 9 star-forming regions that are expected to trace the Carina-Sagittarius arm. Here we present results of G348.70-1.04.

G348.70-1.04 is located in the southern star-forming complex RCW 122. Earlier studies indicated very active star formation, e.g., containing a number of O-type stars (Arnal, et al. 2008). Approximately $20^{\prime}$ north of RCW 122 there is an open cluster Havlen-Moffat 1(HM1) (Havlen \& Moffat 1977), harbouring a group of Of stars (Sanduleak 1974) and two Wolf-Rayet (WR) stars WR87 and WR89. In addition, another WR star, WR91, is located on the south-east edge of RCW 122. Dust emission from this field shows a ring-like structure (Fig. 1), with the open cluster HM1 at the ring's center and RCW 122 projected toward the southern part of the ring.

The spatial arrangement of these young objects is consistent with a triggered starforming scenario between HM1 and RCW 122. However, whether or not they are truly associated is still under debate (Arnal et al. 2008), due to an inconsistency of their distances. For HM1, Havlen \& Moffat (1977) found a spectrosopic distance of $2.9 \pm 0.4$ kpc, and Vázquez \& Baume (2001), using deep UBVRI photomtry, got a distance of 3.3 kpc. The molecular cloud associated with the $12 \mathrm{GHz}$ methanol masers we have observed has an LSR velocity near $13 \mathrm{~km} \mathrm{~s}^{-1}$, corresponding a near kinematic distance of $1.7 \mathrm{kpc}$, while $21 \mathrm{~cm}$ wavelength $\mathrm{H}$ I spectra toward its H II region(s) show absorption features with LSR velocities out to $-44 \mathrm{~km} \mathrm{~s}^{-1}$, suggests a distance of $5 \mathrm{kpc}$ (Radhakrishnan, et al. 1972). Thus the distance to RCW 122 and its relationship to other sources nearby in angle is not well established.

\section{Observations and Results}

We report 8-epoch observations with the VLBA toward the $12 \mathrm{GHz}$ methanol maser source G348.70-1.04 from September 10, 2010 to February 11, 2012. The observing 

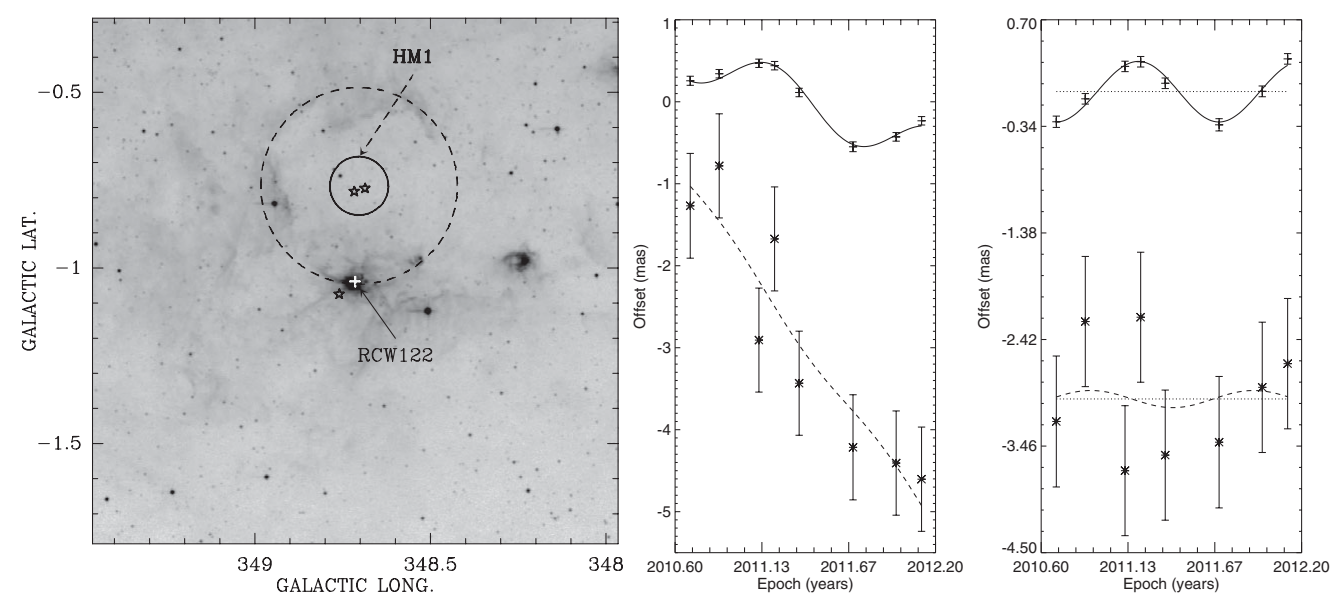

Figure 1. Left panel: MSX $8 \mu \mathrm{m}$ dust emission. The plus-sign denotes the maser, the solid circle denotes the open cluster HM1, and penagrams denote WR stars. The dust emissions shows a ring-like structure (dashed line), with the open cluster HM1 at its center. Parallax fit results: the middle panel shows the eastward and northward position offsets and best fitting model; the right panel is the same as the middle panel, but with the best fit proper motion removed.

dates were chosen to sample the peaks of the parallax signature in right ascension only, as the amplitude of the signature in declination is considerably smaller. Three VLBA calibrators, J1723-3936, J1712-3736 and J1733-3722 (from Immer, Brunthaler, Reid, et al. 2011), were used as background position references.

After calibration, including phase referencing to a maser spot, we imaged the maser and the continuum sources. Only the continuum source nearest to the maser (J1723-3936) was detected, possibly owing to poor phase transfer to the other sources. We estimated the positions of the maser and the background source by fitting elliptical Gaussian brightness distributions to the images. The positions of the maser spots relative to the background source were then used to fit the proper motion and parallax (see the middle and right panel of Fig. 1). Best fit parallax is $0.296 \pm 0.026$ mas, giving a distance of $3.38_{-0.27}^{+0.33}$

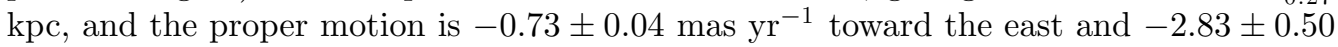
mas $\mathrm{yr}^{-1}$ toward the north.

The trigonometric parallax places the star formation region RCW 122, not in the Carina-Sagittarius arm, but in the Scutum-Centaurus arm. Also, it confirmes the physical connection between the star-forming region RCW 122 and open cluster HM1.

This work was supported by the Chinese NSF through grants NSF 11133008, NSF 11073054, NSF 10621303, and the Key Laboratory for Radio Astronomy, CAS.

\section{References}

Arnal, E. M., Duronea, N. U., \& Tesori, J. C. 2008, AछA, 486, 807

Havlen, R. J. \& Moffat, A. F. J. 1977, A\& A, 58, 351

Immer, K., Brunthaler, A., \& Reid, M. J., et al. 2011, ApJS, 194, 25

Radhakrishnan, V., Goss, W. M., Murray, J. D., \& Brooks, J. W. 1972, ApJS, 24, 49

Sanduleak, N 1974, PASP, 86, 461

Vázquez, R. A., \& Baume, G. A\& A, 371, 908 